\title{
OPEN Liver MRI and clinical findings to predict response after drug eluting bead transarterial chemoembolization in hepatocellular carcinoma
}

\author{
Jeong Yeop Lee ${ }^{1}$, Byung Chan Lee ${ }^{1}$, Hyoung Ook Kim², Suk Hee Heo ${ }^{1,3}$, Sang Soo $\mathrm{Shin}^{2,3}$ \& \\ Yong Yeon Jeong ${ }^{1,3 凶}$
}

To identify the gadoxetic acid (GA)-enhanced magnetic resonance imaging (MRI) and laboratory findings that enable prediction of treatment response and disease-free survival (DFS) after the first session of drug eluting bead transarterial chemoembolization (DEB-TACE) in patients with hepatocellular carcinoma (HCC). A total of 55 patients who underwent GA-enhanced MRI and DEBTACE from January 2014 to December 2018 were included. All MRI features were reviewed by two radiologists. Treatment response was evaluated according to the modified Response Evaluation Criteria in Solid Tumors. Univariate and multivariate logistic regression analyses were used to determine predictive factors of treatment response and DFS, respectively. A total of 27 patients (49.1\%) achieved complete response (CR) after one session of treatment. There were no significant differences between the two groups in terms of clinical and laboratory characteristics. Heterogeneous signal intensity in the hepatobiliary phase (HBP) was the only independent predictor of non-CR (odds ratio, $4.807 ; p=0.048)$. Recurrent HCC was detected in 19 patients $(70.4 \%)$ after $C R$. In the multivariate analysis, elevated serum alpha-fetoprotein (AFP) level $(\geq 30 \mathrm{ng} / \mathrm{mL}$ ) was the only significant parameter associated with DFS (hazard ratio, $2.916 ; p=0.040$ ). This preliminary study demonstrated that heterogeneous signal intensity in the HBP and high serum AFP were useful predictive factors for poor treatment response and short DFS after DEB-TACE, respectively.

Hepatocellular carcinoma (HCC) is the sixth most common type of cancer and was the fourth leading cause of cancer-related death worldwide in $2018^{1}$. Treatment options for HCC depend on the stage at the time of diagnosis. Surgical resection, liver transplantation, and radiofrequency ablation (RFA) are curative treatments for HCC. However, these therapeutic options are only appropriate for very early or early stage $\mathrm{HCC}^{2-4}$, and only about $30-40 \%$ of patients are diagnosed in these stages ${ }^{5}$. Transarterial chemoembolization (TACE) is a secondary procedure that can be effective for cases in which curative treatment is not an option, and has been established as the standard treatment for intermediate stage $\mathrm{HCC}^{2}$. TACE is also used for slowing the progression of cancer in patients waiting for liver transplantation ${ }^{6}$. Moreover, it can be an alternative therapeutic option when RFA is not possible due to the location of the lesion or when surgery is restricted due to other underlying diseases ${ }^{7}$.

TACE with drug eluting beads (DEB-TACE) is a subtype of TACE ${ }^{8}$. For this technique, chemotherapeutic agents are contained in a microsphere and gradually released within the tumor. This process allows greater and prolonged drug retention in HCC and lower release of the drug into the systemic circulation. Several studies have reported that peak serum concentrations of doxorubicin are significantly lower after DEB-TACE than after conventional TACE ${ }^{8,9}$, resulting in fewer systemic complications ${ }^{9,10}$. DEB-TACE is currently being used in practice, but two large prospective randomized trials have not proven supremacy of DEB-TACE over conventional TACE in the treatment of $\mathrm{HCC}^{10,11}$.

${ }^{1}$ Department of Radiology, Chonnam National University Hwasun Hospital, 322 Seoyang-ro, Hwasun 58128, Korea. ${ }^{2}$ Department of Radiology, Chonnam National University Hospital, 42 Jebong-ro, Dong-gu, Gwangju 61469, Korea. ${ }^{3}$ Department of Radiology, Chonnam National University Medical School, 42 Jebong-ro, Dong-gu, Gwangju 61469, Korea. ${ }^{\boxplus e m a i l}$ : yjeong@jnu.ac.kr 


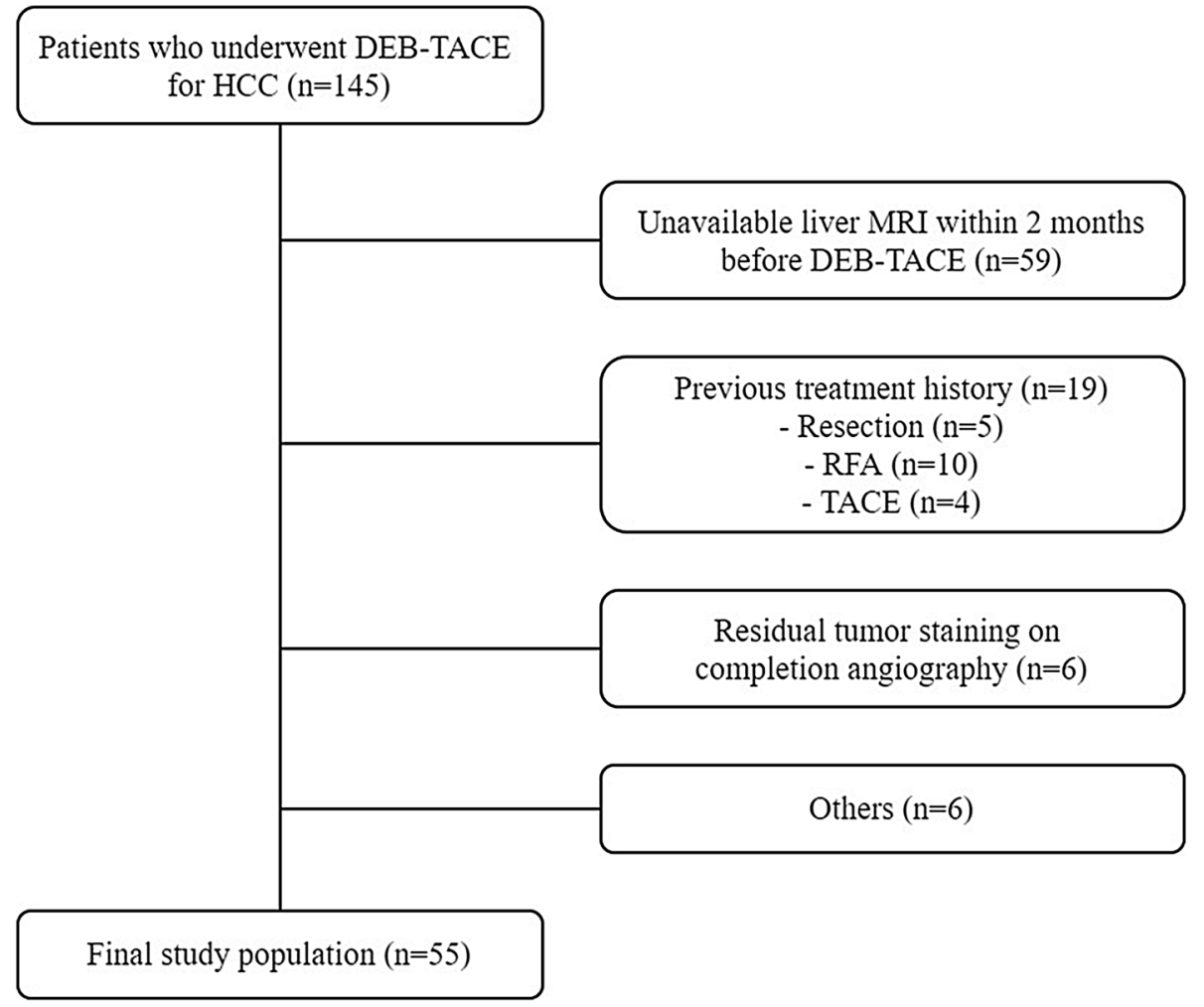

Figure 1. Flow chart of the study population. DEB-TACE drug eluting bead transarterial chemoembolization, $H C C$ hepatocellular carcinoma, MRI magnetic resonance imaging, $R F A$ radiofrequency ablation.

Follow-up computed tomography (CT) and magnetic resonance imaging (MRI) after TACE are useful for diagnosing and evaluating the response. Gadoxetic acid (GA)-enhanced liver MRI has been widely used to evaluate HCC due to its excellent diagnostic performance. For patients with HCC, prognosis is strongly associated with the degree of response to the first TACE ${ }^{12,13}$. Therefore, predicting the response to TACE based on pretreatment MRIs could be used for establishing a treatment strategy. Recently, several studies have investigated pretreatment imaging findings, including MRIs, which may be predictors of treatment response or overall outcomes in conventional TACE ${ }^{14-18}$. However, there are fewer studies about DEB-TACE than conventional TACE. Some studies have mentioned the MRI findings in DEB-TACE, but they focused on angiographic characteristics rather than specific MRI findings ${ }^{19,20}$ or evaluated only posttreatment MRI $^{21}$. Our study aimed to identify pretreatment GA-enhanced MRI findings that may enable the prediction of treatment response and disease-free survival (DFS) after the first session of DEB-TACE in patients with HCC.

\section{Methods}

Patients. We retrospectively analyzed the patient database at a single tertiary hospital (Chonnam National University Hwasun Hospital, Hwasun, Korea). The Institutional Review Board of Chonnam National University Hwasun Hospital approved this study and waived informed consent requirement due to the retrospective study design. All methods were carried out in accordance with relevant guidelines and regulations. From January 2014 to December 2018, 145 consecutive patients underwent DEB-TACE for HCC at our institution. HCC diagnosis was based on imaging following the American Association for the Study of Liver Diseases (AASLD) criteria $^{3}$. In our study, MRI was used for all HCC diagnoses. The exclusion criteria for our study were as follows: (a) no available GA-enhancement liver MRI within 2 months of DEB-TACE; (b) history of previous treatment for HCC, including surgical resection, RFA, TACE, or other therapies; (c) residual tumor staining on completion angiography during TACE; (d) other concurrent malignancies; and (e) insufficient follow-up examination. Ultimately, 55 patients were enrolled in the study (Fig. 1).

The following clinical information, laboratory and procedural factors that could have potentially influenced the therapeutic response were analyzed: age, sex, etiology of liver disease, presence of liver cirrhosis, Child-Pugh class, serum alpha-fetoprotein (AFP), protein induced by vitamin $\mathrm{K}$ absence or antagonist-II (PIVKA-II), and DEB particles size.

MRI techniques. A 3T MR scanner (MAGNETOM Skyra; Siemens Healthineers) with an 18-channel body array coil was used for all imaging according to the specified protocols (Table 1). Breath-hold axial in- and opposed-phase T1-weighted images (T1WI) were obtained using the two-dimensional volumetric interpolated breath-hold examination (VIBE) technique. Breath-hold T2-weighted images (T2WI) were obtained using the 


\begin{tabular}{|l|l|l|l|}
\hline & In- \& opposed-phase T1WI & T2WI \& heavily T2WI & Dynamic contrast enhancement \\
\hline Pulse sequence & VIBE & HASTE & VIBE \\
\hline TR/TE $(\mathrm{ms})$ & $4.4 / 1.4,2.3$ & $2000 / 81,166$ & $3.5 / 1.3$ \\
\hline Flip angle $\left(^{\circ}\right)$ & 9 & $90-150$ & 13 \\
\hline Section thickness $(\mathrm{mm})$ & 3 & 5 & 3 \\
\hline Interslice gap $(\mathrm{mm})$ & 0 & 0.5 & 0 \\
\hline Field of view $(\mathrm{mm})$ & $300-400$ & $300-400$ & $300-400$ \\
\hline Matrix number & $320 \times 256$ & $320 \times 256$ & $352 \times 194$ \\
\hline
\end{tabular}

Table 1. Imaging parameters for the MRI protocols. HASTE half-Fourier acquired single-shot turbo spin echo, T1WI T1-weighted image, T2WI T2-weighted image, TE echo time, TR repetition time, VIBE volumetric interpolated breath-hold examination.

fat-suppressed half-Fourier acquisition single-shot turbo-spin echo (HASTE) technique, and heavily T2WI was obtained using the breath-hold HASTE sequence without fat suppression. For contrast-enhanced dynamic T1WI using the $3 \mathrm{D}$ VIBE technique, $0.1 \mathrm{~mL} / \mathrm{kg}(0.25 \mathrm{mmol} / \mathrm{mL})$ of $\mathrm{GA}$ (Primovist ${ }^{\oplus}$, Bayer Healthcare) was injected with an MRI-compatible injector (Nemoto, Kyorindo) at a flow rate of $1 \mathrm{~mL} / \mathrm{sec}$ and followed by $20 \mathrm{~mL}$ of $0.9 \%$ saline flush. Axial images were acquired at 30,60, 120, 180,600, and $900 \mathrm{~s}$ after injection of the contrast agent, and imaging at $900 \mathrm{~s} \mathrm{(15} \mathrm{min)} \mathrm{was} \mathrm{considered} \mathrm{the} \mathrm{hepatobiliary} \mathrm{phase} \mathrm{(HBP).} \mathrm{Additionally,} \mathrm{contrast-enhanced}$ coronal images were obtained at $5 \mathrm{~min}$.

Pretreatment MRI analysis. All GA-enhanced MR images were reviewed by two abdominal radiologists (with 25 and 15 years of experience) who were blinded to clinical information and pathological analysis. After the first independent image evaluation, any discrepancies in the results between the two reviewers were resolved by consensus. Firstly, the size, multiplicity, and location of the tumors were estimated. The tumor location was classified into two categories: peripheral or central. Central location was defined as a tumor located within $0.5 \mathrm{~cm}$ of the first or second branches of the portal vein, or a tumor located at least $3 \mathrm{~cm}$ away from the liver capsule $^{14}$. In the case of multiple tumors, only when all tumors were not in the central location, it was regarded as a peripheral tumor location. In our study, all tumors had a nodular appearance and there was no infiltrative type HCC.

The following MR imaging features in each HCC were evaluated: most of these imaging features have been developed based on previous studies ${ }^{22,23}$. In patients with multiple HCC lesions, the largest tumor was selected for assessment.

- Signal heterogeneity in the arterial phase; a nonenhanced area within the tumor in the arterial phase ${ }^{15}$

- Arterial rim enhancement; the presence of irregular ring-like areas of enhancement with a central hypovascular area in the arterial phase

- Arterial peritumoral enhancement; a detectable portion of polygonal or crescent-shaped enhancement outside of the tumor border that became isointense with background liver parenchyma in the later dynamic phase

- Radiologic capsule; a distinct peripheral rim with delayed contrast enhancement involving more than $90 \%$ of the tumor circumference

- Marginal irregularity; a non-smooth margin with a budding portion at the periphery of the tumor protruding into the hepatic parenchyma in the HBP

- Peritumoral hypointensity in the HBP; a wedge-shaped or flame-like area of low signal intensity in the liver parenchyma located outside of the tumor border in the HBP

- Signal heterogeneity in the HBP; a iso- or hyperintense portion was present inside the tumor in the HBP, compared with the background liver ${ }^{24}$

- Grossly visible vascular invasion

- Conspicuous rim; a smooth and discernable peripheral rim of the tumor appearing hypointense or hyperintense on T1WI or T2WI

- Peritumoral hyperintensity on T2WI; a polygonal or wedge-shaped area of high signal intensity outside of the tumor border on T2WI

- Intratumoral fat; an intratumoral area with decreased signal intensity on opposed-phase T1WI compared with in-phase images

- Intratumoral hyperintense portion on T1WI

Chemoembolization protocols. Doxorubicin (Adriamycin, Ildong) and DEB (DC Bead ${ }^{\star}$, Biocompatibles UK) agents were used for the DEB-TACE technique. The procedures were carried out with DEB particles of different sizes ranging from $70-150 \mu \mathrm{m}$ to $300-500 \mu \mathrm{m}$, according to the manufacturer's instructions. The determination of the sizes of DEB particles used in procedures was made by interventional radiologists considering the tumor size. One vial of DEB agent was loaded with $50 \mathrm{mg}$ of doxorubicin solution, and the preparation was suspended in $30 \mathrm{~mL}$ non-ionic iodized contrast agent (Xenetix ${ }^{\oplus}$, Guerbet). The DEB suspension was administered slowly at a rate of approximately $1 \mathrm{~mL} / \mathrm{min}$, to avoid reflux and non-target embolization. The total dosage used for the TACE procedure was determined by the interventional radiologist based on the extent of 
the tumor burden, but the maximum dose per patient was $100 \mathrm{mg}$ of doxorubicin. The goal of embolization was the stasis of arterial blood flow to the tumor. When multiple lesions were present, the same procedure was used for all lesions. If tumor staining remained on completion angiography due to the large size of the tumor, it was excluded from the study.

Follow-up and response evaluation. The initial post-TACE images were obtained using a 64-multidetector CT scanner (Somatom Sensation 64, Siemens Healthineers; and LightSpeed VCT, GE Healthcare) 4 weeks after the TACE. All examinations were analyzed on site and a secondary reading for response evaluation was performed specifically for this study by an abdominal radiologist who was blinded to all clinical information except that all patients had undergone DEB-TACE. Follow-up contrast-enhanced CTs were repeated every 2-3 months.

The tumor response was estimated using the modified Response Evaluation Criteria in Solid Tumors (mRECIST $)^{25}$. For mRECIST, response is based on residual arterial enhancement rather than pure tumor shrinkage measured by the greatest diameter of the lesion. There are four categories of tumor response according to mRECIST: complete response (CR), disappearance of any intratumoral arterial enhancement in all target lesions; partial response (PR), at least a 30\% decrease in the sum of the diameters of the lesions showing arterial enhancement, taking as reference the baseline sum of the diameters of the target lesions; stable disease (SD), any cases that do not qualify for either PR or progressive disease (PD); and PD, at least a $20 \%$ increase in the sum of the diameters of the lesions showing arterial enhancement, taking as reference the smallest sum of the diameters of the target lesions. For our study, all patients were categorized either into the CR or the non-CR group. The non-CR group included patients with PR or SD; there were no cases of PD in this study. Tumor recurrence after $\mathrm{CR}$ was defined as newly-developed arterial enhancement in the non-enhancing portion of the original mass or new lesions that satisfied the AASLD criteria for $\mathrm{HCC}^{3}$ appearing on subsequent follow-up imaging.

Statistical analysis. To compare variables between the CR and non-CR groups, categorical variables (including MR imaging findings) were analyzed using the chi-square or Fisher's exact test. Continuous variables were evaluated using the Mann-Whitney $U$-test. A logistic regression analysis was performed to identify the predictive factors for CR with DEB-TACE. Variables with a $p$ value $<0.05$ in the univariate logistic regression analysis were entered into the multivariate logistic regression analysis to determine the independent predictors. Odds ratios (ORs) with 95\% confidence intervals (CIs) were estimated. The Cox proportional hazard model was used to identify prognostic factors associated with DFS in the CR group. DFS was defined as the interval between the administration of DEB-TACE and follow-up CT imaging that indicated recurrence. Multivariate models were created using variables that were significant in the univariate analysis $(p<0.05)$. Hazard ratios (HRs) with 95\% CIs were calculated for each factor. All statistical analyses were performed using a statistical software package (SPSS version 25.0, IBM Corp.), and significance was defined as $p<0.05$.

\section{Results}

The patients' baseline characteristics are summarized in Table 2. Fifty-five patients were evaluated in our study. Among them, 27 patients achieved CR, 15 achieved PR, and the others showed SD after the first session of DEB-TACE. Forty-three patients (78.2\%) were classified as Child-Pugh class A, one patient from the non-CR group was classified as Child-Pugh class $\mathrm{C}$, and the others were considered Child-Pugh class B. There were no significant differences between the two groups in terms of age, sex, etiology of liver disease, presence of liver cirrhosis, Child-Pugh class, or laboratory findings involving AFP and PIVKA-II $(p>0.05)$. DEB particles of $70-150 \mu \mathrm{m}$ were used for 33 patients $(60.0 \%)$ and that of $100-300 \mu \mathrm{m}$ or $300-500 \mu \mathrm{m}$ were used for others. But the size of DEB particles was not associated with the difference of the treatment response between the CR and non-CR groups $(66.7 \%$ vs. $53.6 \%$; $p=0.322)$.

Regarding MR imaging features, tumor size was significantly different between the groups. The proportion of patients with a tumor size $\geq 5 \mathrm{~cm}$ was smaller in the CR group than in the non-CR group ( $14.8 \% v s .42 .9 \%$; $p=0.037$ ). The median tumor size of all the included patients was $3.1 \mathrm{~cm}$ (range $1.3-8.5 \mathrm{~cm}$ ). Additionally, heterogeneous arterial enhancement and heterogeneous signal intensity in the HBP were significantly greater in the non-CR group than in the CR group ( $55.6 \%$ vs. $82.1 \%$; $p=0.033$ and $18.5 \%$ vs. $60.7 \%$; $p=0.001$, respectively). Eighteen patients had multiple lesions: nine had two lesions, five had three lesions, and the others had four or more lesions. The proportion of patients with the central location was larger in the non-CR group. However, the multiplicity and location of the tumors did not affect the treatment response $(25.9 \% v s .39 .3 \% ; p=0.291$ and $11.1 \%$ vs. $32.1 \% ; p=0.101$, respectively). All the other MRI findings also were not significantly different between the two groups (Table 3).

Logistic regression was performed to determine the predictive factors of non-CR. In the univariate analysis, tumor size, signal heterogeneity in the arterial phase, and signal heterogeneity in the HBP were significantly different between the CR and non-CR groups (Fig. 2). However, in the multivariate analysis, only heterogeneous signal intensity in the $\mathrm{HBP}$ was significantly different between the groups $(\mathrm{OR}=4.807 ; 95 \% \mathrm{CI}=1.011-22.865$; $p=0.048$ ) (Table 4).

Recurrent lesions were detected in 19 of the 27 patients with CR (70.4\%), with a median DFS of 12 months (range, 3-29 months). The Cox proportional hazard model was used to evaluate the DFS after treatment. In the univariate analysis, AFP and peritumoral low signal intensity in the HBP were significant factors related to DFS. After including these variables in the multivariate analysis, the only significant parameter was elevated serum AFP level $(\geq 30 \mathrm{ng} / \mathrm{mL})(\mathrm{HR}=2.916$; 95\% CI 1.048-8.108; $p=0.040)$ (Table 5). 


\begin{tabular}{|l|l|l|l|}
\hline Characteristics & CR $(\mathbf{n}=27)$ & Non-CR (n=28) & $p$ value \\
\hline Age (years) & $67.96 \pm 11.57$ & $65.71 \pm 12.31$ & 0.704 \\
\hline Sex & $21(77.8)$ & $21(75.0)$ & 0.808 \\
\hline Male & $6(22.2)$ & $7(25.0)$ & \\
\hline Female & $16(59.3)$ & $19(67.9)$ & 0.508 \\
\hline Etiology of liver disease & \multicolumn{5}{|l|}{} \\
\hline Viral & $11(40.7)$ & $9(32.1)$ & \\
\hline Non-viral & \multicolumn{5}{|l|}{} \\
\hline Liver cirrhosis & $5(18.5)$ & $6(21.4)$ & 0.787 \\
\hline Absent & $22(81.5)$ & $22(78.6)$ & \\
\hline Present & & & \\
\hline Child-Pugh class & $21(77.8)$ & $22(78.6)$ & 0.943 \\
\hline A & $6(22.2)$ & $6(21.4)$ & \\
\hline B or C & $4.1(2.6-4.7)$ & $3.8(2.7-5.1)$ & 0.448 \\
\hline Albumin (g/dL) & $0.6(0.3-1.9)$ & $0.8(0.3-2.4)$ & 0.427 \\
\hline Total bilirubin (mg/dL) & $1.15(0.97-1.94)$ & $1.12(0.99-1.70)$ & 0.844 \\
\hline Prothrombin time (INR) & & & 0.777 \\
\hline AFP (ng/mL) & $12(44.4)$ & $14(50.0)$ & \\
\hline$<30$ & $14(51.9)$ & $14(50.0)$ & \\
\hline$\geq 30$ & 1 & 0 & \\
\hline NA & $14(51.9)$ & $14(50.0)$ & 0.662 \\
\hline PIVKA-II (mAU/mL) & $11(40.7)$ & $14(50.0)$ & \\
\hline$<100$ & 2 & 0 & \\
\hline$\geq 100$ & & & \\
\hline NA & & & \\
\hline
\end{tabular}

Table 2. Baseline patient characteristics. Data are presented as mean $\pm \mathrm{SD}$, number (\%), or median (range). $A F P$ alpha-fetoprotein, $C R$ complete response, INR international normalized ratio, NA not available, PIVKA-II protein induced by vitamin $\mathrm{K}$ absence or antagonist-II.

\section{Discussion}

In this study, we assessed potential factors for predicting treatment response and outcomes in HCC patients who undergo DEB-TACE. The only independent predisposing factor for non-CR was heterogeneous signal intensity in the HBP, with generally better treatment response for tumors showing homogeneous signal intensity in the HBP. Tumor size and heterogeneous arterial enhancement were significant factors in the univariate analysis but were not independent predictive factors in the multivariate analysis. AFP and peritumoral low signal intensity in the HBP were also significant factors related to DFS in the univariate analysis; however, only elevated serum AFP was an independent prognostic factor associated with shortened DFS in the CR group.

The degree of signal intensity in the HBP using GA-enhanced MR imaging is determined by organic aniontransporting polypeptide 8 (OATP8), since it is responsible for the intracellular uptake of $\mathrm{GA}^{24,26}$. Several studies have noted the role of OATP8 as a drug uptake transporter ${ }^{27,28}$. These studies suggest that OATP8 is involved in the influx of doxorubicin, so HCC with a high expression of OATP8 is likely to respond well to TACE. However, if a single tumor shows heterogeneous signal intensity in the HBP, it means that there are various degrees of tumor differentiation and OATP8 levels coexisting inside the tumor. HCC is well-known to be heterogeneous morphologically and genetically. Heterogeneous signal intensity in the HBP suggests the tumoral heterogeneity, which is associated with drug resistance in HCC and may influence treatment outcomes ${ }^{29}$. Our study showed that heterogeneous signal intensity in the HBP was a predictive factor of poor treatment response in the multivariate analysis.

Fujita et al. ${ }^{30}$ demonstrated that heterogeneous signal intensity in the HBP was associated with worse diseasefree survival after surgical resection than homogeneous hypointensity in the HBP. However, Bae et al. ${ }^{16}$ suggested that heterogeneous signal intensity in the HBP was not associated with the prognosis after surgical resection, RFA, or conventional TACE, which seems to contradict previous findings. In our study, heterogeneous signal intensity in the HBP was not an independent factor for shortened DFS after CR.

Heterogeneous arterial enhancement has been associated with worse treatment response and poor prognosis in conventional TACE and DEB-TACE ${ }^{19,31,32}$. Tumor size is also a well-known predictor related to response and prognosis for conventional TACE ${ }^{12,15,17,18,33-35}$ and DEB-TACE ${ }^{20,31}$. These results can be explained by the degree of tumor differentiation, as a poorly differentiated HCC is more likely to manifest in larger tumor size and heterogeneous arterial enhancement ${ }^{36,37}$. These factors are related to the lower arterial blood supply, which leads to decreased tumor staining during the TACE procedure ${ }^{18,19,38}$. Therefore, doxorubicin is not evenly distributed inside these tumors, reducing the intratumoral drug concentration, and decreasing the therapeutic effect of TACE. Poorly differentiated HCC has been shown to respond poorly to DEB-TACE ${ }^{6}$. Our study showed that heterogeneous arterial enhancement and larger tumor size were significant in the univariate analysis, but not 


\begin{tabular}{|c|c|c|c|}
\hline Characteristics & CR $(n=27)$ & Non-CR $(n=28)$ & $p$ value \\
\hline Tumor size (cm) & & & 0.037 \\
\hline$<5$ & $23(85.2)$ & $16(57.1)$ & \\
\hline$\geq 5$ & $4(14.8)$ & $12(42.9)$ & \\
\hline Tumor number & & & 0.291 \\
\hline Single & $20(74.1)$ & $17(60.7)$ & \\
\hline Multiple & $7(25.9)$ & $11(39.3)$ & \\
\hline Tumor location & & & 0.101 \\
\hline Peripheral & $24(88.9)$ & $19(67.9)$ & \\
\hline Central & $3(11.1)$ & $9(32.1)$ & \\
\hline Heterogeneity on AP & & & 0.033 \\
\hline Homogeneous & $12(44.4)$ & $5(17.9)$ & \\
\hline Heterogeneous & $15(55.6)$ & $23(82.1)$ & \\
\hline Rim enhancement on AP & & & 0.205 \\
\hline Absent & $19(70.4)$ & $24(85.7)$ & \\
\hline Present & $8(29.6)$ & $4(14.3)$ & \\
\hline Peritumoral enhancement on AP & & & 0.702 \\
\hline Absent & $18(66.7)$ & $20(71.4)$ & \\
\hline Present & $9(33.3)$ & $8(28.6)$ & \\
\hline Radiologic capsule & & & 0.937 \\
\hline Absent & $20(74.1)$ & $21(75.0)$ & \\
\hline Present & $7(25.9)$ & $7(25.0)$ & \\
\hline Marginal irregularity & & & 0.349 \\
\hline Absent & $14(51.9)$ & $11(39.3)$ & \\
\hline Present & $13(48.1)$ & $17(60.7)$ & $>0.999$ \\
\hline \multicolumn{4}{|l|}{ Peritumoral low SI on HBP } \\
\hline Absent & $23(85.2)$ & $23(82.1)$ & \\
\hline Present & $4(14.8)$ & $5(17.9)$ & \\
\hline Heterogeneity on HBP & & & 0.001 \\
\hline Homogeneous & $22(81.5)$ & $11(39.3)$ & \\
\hline Heterogeneous & $5(18.5)$ & $17(60.7)$ & \\
\hline Gross vascular invasion & & & 0.611 \\
\hline Absent & $26(96.3)$ & $25(89.3)$ & \\
\hline Present & $1(3.7)$ & $3(10.7)$ & \\
\hline Conspicuous rim & & & 0.660 \\
\hline Absent & $17(63.0)$ & $16(57.1)$ & \\
\hline Present & $10(37.0)$ & $12(42.9)$ & \\
\hline Peritumoral high SI on T2WI & & & 0.503 \\
\hline Absent & $21(77.8)$ & $24(85.7)$ & \\
\hline Present & $6(22.2)$ & $4(14.3)$ & \\
\hline Intratumoral fat & & & 0.246 \\
\hline Absent & $22(81.5)$ & $19(67.9)$ & \\
\hline Present & $5(18.5)$ & $9(32.1)$ & \\
\hline Intratumoral high SI on T1WI & & & 0.589 \\
\hline Absent & $21(77.8)$ & $20(71.4)$ & \\
\hline Present & $6(22.2)$ & $8(28.6)$ & \\
\hline
\end{tabular}

Table 3. MRI features of target lesions. $A P$ arterial phase, $C R$ complete response, $H B P$ hepatobiliary phase, $S I$ signal intensity, T1WI T1-weighted image, T2WI T2-weighted image.

independent risk factors of treatment response in the multivariate analysis. Further investigations are needed to determine the correlations among tumor size, heterogeneous arterial enhancement, and treatment outcomes.

Peritumoral hypointensity in the HBP has already been reported as a major factor related to microvascular invasion ${ }^{16,17}$. Furthermore, previous studies have suggested that microvascular invasion is a predictor of early recurrence after surgical resection of $\mathrm{HCC}^{23,39}$. One possible explanation for this is the change in peritumoral perfusion resulting from microvascular invasion, which may influence OATPs, reducing the uptake of GA in hepatocytes around the tumor ${ }^{23}$. Two recent studies have investigated the predictive value of peritumoral hypointensity in the HBP for response to conventional TACE and survival, but they showed contradictory results ${ }^{16,17}$. In our study, peritumoral hypointensity in the HBP was not an independent factor for shortened DFS. Therefore, 

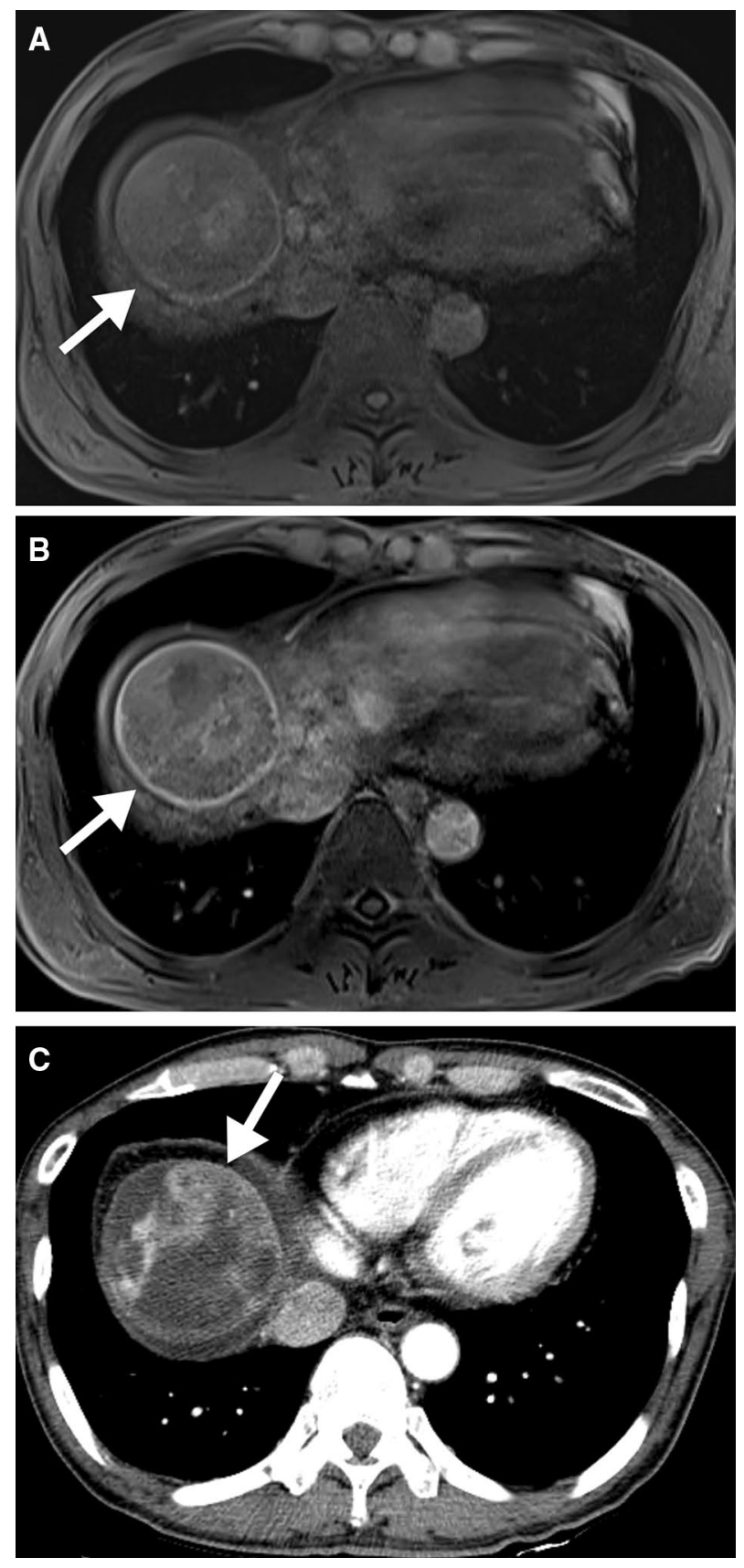

Figure 2. A 50-year-old man with hepatocellular carcinoma in hepatic dome (arrows). Gadoxetic acidenhanced MR images show hypointensity in the precontrast scan (A) and heterogeneous signal intensity in the hepatobiliary phase (B). Residual tumor with arterial enhancement remains on a follow-up CT taken 27 days after DEB-TACE (C). Treatment response was determined to be a stable disease. DEB-TACE drug eluting bead transarterial chemoembolization, $C T$ computed tomography, $M R$ magnetic resonance.

further studies on peritumoral hypointensity in the HBP and its effect on the prognosis of patients with HCC after DEB-TACE are necessary.

High serum AFP has been found to be a risk factor for incomplete response, early recurrence, and poor outcomes after TACE ${ }^{32-35,40}$. However, only one of these studies focused on DEB-TACE specifically ${ }^{40}$, and a multivariate analysis was not performed in that study. Generally, high levels of serum AFP in HCC are associated with a worse differentiation grade than low levels of serum AFP. In addition, decreased serum AFP levels after TACE is considered a positive response to treatment and is also used as a predictor of prognosis ${ }^{34}$. In our multivariate analysis, we found that elevated serum AFP $(\geq 30 \mathrm{ng} / \mathrm{mL})$ was the only independent factor predicting shorter DFS after CR.

There were several limitations to our study. First, it was a retrospective study design of a single center. Second, the patient population was relatively small. However, to our knowledge, this is the first study about the 


\begin{tabular}{|l|l|l|l|l|l|l|}
\hline \multirow{2}{*}{ Variable } & \multicolumn{3}{|l|}{ Univariate analysis } & \multicolumn{3}{l|}{ Multivariate analysis } \\
\hline & OR & $\mathbf{9 5 \%}$ CI & \multicolumn{1}{l}{$\boldsymbol{p}$ value } & OR & $\mathbf{9 5 \%}$ CI & $\boldsymbol{p}$ value \\
\hline Age & 0.984 & $0.940-1.029$ & 0.481 & & & \\
\hline Sex (male) & 0.857 & $0.246-2.983$ & 0.809 & & & \\
\hline Etiology (viral) & 1.451 & $0.481-4.377$ & 0.508 & & & \\
\hline Liver cirrhosis & 0.833 & $0.221-3.138$ & 0.788 & & & \\
\hline Child-Pugh class (B or C) & 0.955 & $0.265-3.433$ & 0.943 & & & \\
\hline AFP ( $\geq 30$ ng/mL) & 0.857 & $0.294-2.497$ & 0.778 & & & \\
\hline PIVKA-II ( $\geq 100$ mAU/mL) & 1.273 & $0.431-3.758$ & 0.662 & & & \\
\hline Tumor size ( $\geq 5$ cm) & 4.312 & $1.176-15.809$ & 0.027 & 1.286 & $0.233-7.081$ & 0.773 \\
\hline Multiple tumors & 1.849 & $0.587-5.822$ & 0.294 & & & \\
\hline Central tumor location & 3.789 & $0.899-15.972$ & 0.070 & & & \\
\hline Heterogeneity on AP & 3.680 & $1.076-12.583$ & 0.038 & 1.663 & $0.401-6.888$ & 0.483 \\
\hline Rim enhancement on AP & 0.396 & $0.103-1.516$ & 0.176 & & & \\
\hline Peritumoral enhancement on AP & 0.800 & $0.254-2.516$ & 0.703 & & & \\
\hline Radiologic capsule & 0.952 & $0.283-3.205$ & 0.937 & & & \\
\hline Marginal irregularity & 1.664 & $0.571-4.853$ & 0.351 & & & \\
\hline Peritumoral low SI on HBP & 1.250 & $0.297-5.256$ & 0.761 & & & \\
\hline Heterogeneity on HBP & 6.800 & $1.983-23.314$ & 0.002 & 4.807 & $1.011-22.865$ & 0.048 \\
\hline Gross vascular invasion & 3.120 & $0.304-32.029$ & 0.338 & & & \\
\hline Conspicuous rim & 1.275 & $0.432-3.761$ & 0.660 & & & \\
\hline Peritumoral high SI on T2WI & 0.583 & $0.145-2.352$ & 0.449 & & & \\
\hline Intratumoral fat & 2.084 & $0.595-7.302$ & 0.251 & & & \\
\hline Intratumoral high SI on T1WI & 1.400 & $0.412-4.756$ & 0.590 & & & \\
\hline
\end{tabular}

Table 4. Logistic regression analysis of predictive factors for non-complete response. AFP alpha-fetoprotein, $A P$ arterial phase, $C I$ confidence interval, $H B P$ hepatobiliary phase, OR odds ratio, PIVKA-II protein induced by vitamin $\mathrm{K}$ absence or antagonist-II, SI signal intensity, T1WI T1-weighted image, T2WI T2-weighted image.

\begin{tabular}{|l|l|l|l|l|l|l|}
\hline \multirow{2}{*}{ Variable } & \multicolumn{3}{ll}{ Univariate analysis } & \multicolumn{2}{l|}{ Multivariate analysis } \\
\cline { 2 - 7 } & HR & $\mathbf{9 5 \%}$ CI & $\boldsymbol{p}$ value & HR & 95\% CI & $\boldsymbol{p}$ value \\
\hline Sex (male) & 0.927 & $0.306-2.809$ & 0.893 & & & \\
\hline Etiology (viral) & 2.324 & $0.833-6.485$ & 0.107 & & & \\
\hline Child-Pugh class (B or C) & 1.086 & $0.384-3.069$ & 0.131 & & & \\
\hline AFP ( $\geq 30$ ng/mL) & 3.415 & $1.293-9.016$ & 0.013 & 2.916 & $1.048-8.108$ & 0.040 \\
\hline PIVKA-II ( $\geq 100$ mAU/mL) & 1.308 & $0.513-3.336$ & 0.574 & & & \\
\hline Tumor size ( $\geq 5$ cm) & 0.507 & $0.116-2.206$ & 0.365 & & & \\
\hline Multiple tumors & 2.173 & $0.811-5.824$ & 0.123 & & & \\
\hline Central tumor location & 0.251 & $0.033-1.894$ & 0.180 & & & \\
\hline Heterogeneity on AP & 0.789 & $0.319-1.950$ & 0.608 & & & \\
\hline Rim enhancement on AP & 0.843 & $0.302-2.349$ & 0.744 & & & \\
\hline Peritumoral enhancement on AP & 0.866 & $0.328-2.288$ & 0.771 & & & \\
\hline Radiologic capsule & 0.706 & $0.234-2.133$ & 0.537 & & & \\
\hline Marginal irregularity & 1.891 & $0.752-4.756$ & 0.176 & & & \\
\hline Peritumoral low SI on HBP & 4.143 & $1.236-13.887$ & 0.021 & 2.488 & $0.711-8.710$ & 0.154 \\
\hline Heterogeneity on HBP & 0.158 & $0.021-1.200$ & 0.075 & & & \\
\hline Gross vascular invasion & 4.700 & $0.548-40.281$ & 0.158 & & & \\
\hline Conspicuous rim & 0.818 & $0.309-2.166$ & 0.686 & & & \\
\hline Peritumoral high SI on T2WI & 1.698 & $0.609-4.736$ & 0.312 & & & \\
\hline Intratumoral fat & 0.902 & $0.298-2.729$ & 0.855 & & & \\
\hline Intratumoral high SI on T1WI & 0.620 & $0.180-2.139$ & 0.450 & & & \\
\hline
\end{tabular}

Table 5. Predictive factors for disease-free survival after complete response. $A F P$ alpha-fetoprotein, $A P$ arterial phase, $C I$ confidence interval, $H B P$ hepatobiliary phase, $H R$ hazard ratio, PIVKA-II protein induced by vitamin $\mathrm{K}$ absence or antagonist-II, SI signal intensity, T1WI T1-weighted image, T2 WI T2-weighted image. 
correlation of pretreatment GA-enhanced MRI findings with DEB-TACE results. A study including a larger number of patients would be necessary for further validation of these results. Third, there was a discrepancy in the size of DEB particles between the patients, which may be a confounding factor since smaller DEB particles were shown to have better efficacy in some previous studies ${ }^{20,21}$. However, in our study, the particle size was not significant factor for treatment response.

In conclusion, heterogeneous signal intensity in the HBP on GA-enhanced MRI may be a helpful biomarker to predict non-CR after DEB-TACE in patients with HCC. And elevated serum AFP was a valuable factor for predicting shorter DFS after DEB-TACE.

Received: 26 April 2021; Accepted: 1 November 2021

Published online: 15 December 2021

\section{References}

1. Bray, F. et al. Global cancer statistics 2018: GLOBOCAN estimates of incidence and mortality worldwide for 36 cancers in 185 countries. CA Cancer J. Clin. 68, 394-424. https://doi.org/10.3322/caac.21492 (2018).

2. European Association for the Study of the Liver. Electronic address, e. e. e. \& European Association for the Study of the, L. EASL Clinical Practice Guidelines: Management of hepatocellular carcinoma. J. Hepatol. 69, 182-236. https://doi.org/10.1016/j.jhep. 2018.03.019 (2018).

3. Bruix, J., Sherman, M. \& American Association for the Study of Liver, D. Management of hepatocellular carcinoma: An update. Hepatology 53, 1020-1022. https://doi.org/10.1002/hep.24199 (2011).

4. Marrero, J. A. et al. Diagnosis, staging, and management of hepatocellular carcinoma: 2018 practice guidance by the American Association for the Study of Liver Diseases. Hepatology 68, 723-750. https://doi.org/10.1002/hep.29913 (2018).

5. Park, J. W. et al. Global patterns of hepatocellular carcinoma management from diagnosis to death: The BRIDGE Study. Liver Int 35, 2155-2166. https://doi.org/10.1111/liv.12818 (2015).

6. Sandow, T. A. et al. Assessment of response to transcatheter arterial chemoembolization with doxorubicin-eluting microspheres: Tumor biology and hepatocellular carcinoma recurrence in a 5-year transplant cohort. Radiology 286, 1072-1083. https://doi.org/ 10.1148/radiol.2017170731 (2018).

7. Yang, H. J. et al. Small single-nodule hepatocellular carcinoma: Comparison of transarterial chemoembolization, radiofrequency ablation, and hepatic resection by using inverse probability weighting. Radiology 271, 909-918. https://doi.org/10.1148/radiol. $13131760(2014)$

8. Varela, M. et al. Chemoembolization of hepatocellular carcinoma with drug eluting beads: Efficacy and doxorubicin pharmacokinetics. J. Hepatol. 46, 474-481. https://doi.org/10.1016/j.jhep.2006.10.020 (2007).

9. Poon, R. T. et al. A phase I/II trial of chemoembolization for hepatocellular carcinoma using a novel intra-arterial drug-eluting bead. Clin. Gastroenterol. Hepatol. 5, 1100-1108. https://doi.org/10.1016/j.cgh.2007.04.021 (2007).

10. Lammer, J. et al. Prospective randomized study of doxorubicin-eluting-bead embolization in the treatment of hepatocellular carcinoma: Results of the PRECISION V study. Cardiovasc. Intervent. Radiol. 33, 41-52. https://doi.org/10.1007/s00270-009-9711-7 (2010).

11. Golfieri, R. et al. Randomised controlled trial of doxorubicin-eluting beads vs conventional chemoembolisation for hepatocellular carcinoma. Br. J. Cancer 111, 255-264. https://doi.org/10.1038/bjc.2014.199 (2014).

12. Kim, B. K. et al. Complete response at first chemoembolization is still the most robust predictor for favorable outcome in hepatocellular carcinoma. J. Hepatol. 62, 1304-1310. https://doi.org/10.1016/j.jhep.2015.01.022 (2015).

13. Prajapati, H. J. et al. mRECIST and EASL responses at early time point by contrast-enhanced dynamic MRI predict survival in patients with unresectable hepatocellular carcinoma (HCC) treated by doxorubicin drug-eluting beads transarterial chemoembolization (DEB TACE). Ann. Oncol. 24, 965-973. https://doi.org/10.1093/annonc/mds605 (2013).

14. Park, C. et al. Imaging predictors of survival in patients with single small hepatocellular carcinoma treated with transarterial chemoembolization. Korean J. Radiol. 22, 213-224. https://doi.org/10.3348/kjr.2020.0325 (2021).

15. Tao, P. Y., Zhang, Z. S., Wang, T. C., Yu, M. Q. \& Xiao, Y. D. A predictive model of incomplete response after transarterial chemoembolization for early or intermediate stage of hepatocellular carcinoma: Consideration of hepatic angiographic and cross-sectional imaging. Abdom. Radiol. (NY) 46, 581-589. https://doi.org/10.1007/s00261-020-02701-5 (2021).

16. Bae, J. S., Kim, J. H., Lee, D. H., Kim, J. H. \& Han, J. K. Hepatobiliary phase of gadoxetic acid-enhanced MRI in patients with HCC: Prognostic features before resection, ablation, or TACE. Eur. Radiol. https://doi.org/10.1007/s00330-020-07499-w (2020).

17. Byun, J. et al. Prediction of transarterial chemoembolization refractoriness in patients with hepatocellular carcinoma using imaging features of gadoxetic acid-enhanced magnetic resonance imaging. Acta Radiol. https://doi.org/10.1177/0284185120971844 (2020).

18. Kim, Y. J., Lee, M. H., Choi, S. Y., Yi, B. H. \& Lee, H. K. Magnetic resonance imaging features predictive of an incomplete response to transarterial chemoembolization in patients with hepatocellular carcinoma: A STROBE-compliant study. Medicine 98, e15592. https://doi.org/10.1097/MD.0000000000015592 (2019).

19. Chang, K. H. et al. Predictive imaging for tumor response to drug-eluting microsphere transarterial chemoembolization in patients with BCLC-C advanced hepatocellular carcinoma. Sci. Rep. 9, 20032. https://doi.org/10.1038/s41598-019-56545-1 (2019).

20. Vesselle, G. et al. Predictive factors for complete response of chemoembolization with drug-eluting beads (DEB-TACE) for hepatocellular carcinoma. Eur. Radiol. 26, 1640-1648. https://doi.org/10.1007/s00330-015-3982-y (2016).

21. Lee, S., Kim, K. A., Park, M. S. \& Choi, S. Y. MRI findings and prediction of time to progression of patients with hepatocellular carcinoma treated with drug-eluting bead transcatheter arterial chemoembolization. J. Korean Med. Sci. 30, 965-973. https://doi. org/10.3346/jkms.2015.30.7.965 (2015).

22. An, C. et al. Single hepatocellular carcinoma: Preoperative MR imaging to predict early recurrence after curative resection. Radiology 276, 433-443. https://doi.org/10.1148/radiol.15142394 (2015).

23. Lee, S., Kim, S. H., Lee, J. E., Sinn, D. H. \& Park, C. K. Preoperative gadoxetic acid-enhanced MRI for predicting microvascular invasion in patients with single hepatocellular carcinoma. J. Hepatol. 67, 526-534. https://doi.org/10.1016/j.jhep.2017.04.024 (2017).

24. Kitao, A. et al. Hepatocellular carcinoma: Signal intensity at gadoxetic acid-enhanced MR Imaging-correlation with molecular transporters and histopathologic features. Radiology 256, 817-826. https://doi.org/10.1148/radiol.10092214 (2010).

25. Lencioni, R. \& Llovet, J. M. Modified RECIST (mRECIST) assessment for hepatocellular carcinoma. Semin. Liver Dis. 30, 52-60. https://doi.org/10.1055/s-0030-1247132 (2010).

26. Tsuboyama, T. et al. Hepatocellular carcinoma: Hepatocyte-selective enhancement at gadoxetic acid-enhanced MR imaging-correlation with expression of sinusoidal and canalicular transporters and bile accumulation. Radiology 255, 824-833. https://doi. org/10.1148/radiol.10091557 (2010).

27. Thakkar, N., Lockhart, A. C. \& Lee, W. Role of organic anion-transporting polypeptides (OATPs) in cancer therapy. AAPS J 17, 535-545. https://doi.org/10.1208/s12248-015-9740-x (2015). 
28. Kim, J. W. et al. The value of paradoxical uptake of hepatocellular carcinoma on the hepatobiliary phase of gadoxetic acid-enhanced liver magnetic resonance imaging for the prediction of lipiodol uptake after transcatheter arterial chemoembolization. Eur. J. Radiol. 89, 169-176. https://doi.org/10.1016/j.ejrad.2017.02.004 (2017).

29. Craig, A. J., Labgaa, I., Villacorta-Martin, C., Ningarhari, M. \& Villanueva, A. Tumor heterogeneity and resistance to targeted therapies in hepatocellular carcinoma. In Resistance to Molecular Therapies for Hepatocellular Carcinoma (ed. Villanueva, A.) 1-24 (Springer, 2017).

30. Fujita, N. et al. Hepatocellular carcinoma: Clinical significance of signal heterogeneity in the hepatobiliary phase of gadoxetic acid-enhanced MR imaging. Eur. Radiol. 25, 211-220. https://doi.org/10.1007/s00330-014-3349-9 (2015).

31. Reis, S. P. et al. Tumor enhancement and heterogeneity are associated with treatment response to drug-eluting bead chemoembolization for hepatocellular carcinoma. J. Comput. Assist. Tomogr. 41, 289-293. https://doi.org/10.1097/RCT.0000000000000509 (2017).

32. Saito, N. et al. Transarterial- chemoembolization remains an effective therapy for intermediate-stage hepatocellular carcinoma with preserved liver function. Hepatol. Res. 50, 1176-1185. https://doi.org/10.1111/hepr.13550 (2020).

33. $\mathrm{Hu}, \mathrm{H}$. T. et al. Chemoembolization for hepatocellular carcinoma: Multivariate analysis of predicting factors for tumor response and survival in a 362-patient cohort. J. Vasc. Interv. Radiol. 22, 917-923. https://doi.org/10.1016/j.jvir.2011.03.005 (2011).

34. Jeong, S. O. et al. Predictive factors for complete response and recurrence after transarterial chemoembolization in hepatocellular carcinoma. Gut Liver 11, 409-416. https://doi.org/10.5009/gnl16001 (2017).

35. Rou, W. S. et al. Risk factors and therapeutic results of early local recurrence after transcatheter arterial chemoembolization. World J. Gastroenterol. 20, 6995-7004. https://doi.org/10.3748/wjg.v20.i22.6995 (2014).

36. Kawamura, Y. et al. New classification of dynamic computed tomography images predictive of malignant characteristics of hepatocellular carcinoma. Hepatol. Res. 40, 1006-1014. https://doi.org/10.1111/j.1872-034X.2010.00703.x (2010).

37. Lee, J. H. et al. Enhancement patterns of hepatocellular carcinomas on multiphasic multidetector row CT: Comparison with pathological differentiation. Br. J. Radiol. 85, e573-583. https://doi.org/10.1259/bjr/86767895 (2012)

38. Asayama, Y. et al. Poorly versus moderately differentiated hepatocellular carcinoma: Vascularity assessment by computed tomographic hepatic angiography in correlation with histologically counted number of unpaired arteries. J. Comput. Assist. Tomogr. 31, 188-192. https://doi.org/10.1097/01.rct.0000236417.82395.57 (2007).

39. Cha, D. I. et al. Preoperative prediction for early recurrence can be as accurate as postoperative assessment in single hepatocellular carcinoma patients. Korean J. Radiol. 21, 402-412. https://doi.org/10.3348/kjr.2019.0538 (2020).

40. Dhanasekaran, R. et al. Prognostic factors for survival in patients with unresectable hepatocellular carcinoma undergoing chemoembolization with doxorubicin drug-eluting beads: A preliminary study. HPB (Oxford) 12, 174-180. https://doi.org/10.1111/j. 1477-2574.2009.00138.x (2010).

\section{Acknowledgements}

This research was supported by the Bio \& Medical Technology Development Program of the National Research Foundation (NRF) funded by the Korean government (MSIT) (No. 2019M3E5D1A02068082).

\section{Author contributions}

J.Y.L and Y.Y.J designed the research; all authors performed data collection, analyzed the data, and wrote or reviewed the manuscript.

\section{Competing interests}

The authors declare no competing interests.

\section{Additional information}

Correspondence and requests for materials should be addressed to Y.Y.J.

Reprints and permissions information is available at www.nature.com/reprints.

Publisher's note Springer Nature remains neutral with regard to jurisdictional claims in published maps and institutional affiliations.

(c) Open Access This article is licensed under a Creative Commons Attribution 4.0 International License, which permits use, sharing, adaptation, distribution and reproduction in any medium or format, as long as you give appropriate credit to the original author(s) and the source, provide a link to the Creative Commons licence, and indicate if changes were made. The images or other third party material in this article are included in the article's Creative Commons licence, unless indicated otherwise in a credit line to the material. If material is not included in the article's Creative Commons licence and your intended use is not permitted by statutory regulation or exceeds the permitted use, you will need to obtain permission directly from the copyright holder. To view a copy of this licence, visit http://creativecommons.org/licenses/by/4.0/.

(C) The Author(s) 2021 